\title{
Zwei seltene operativ geheilte Gehirnerkrankungen (1. Hirngummi bei Lues hereditaria tarda, 2. Metastatischer Hirnabsceß).
}

\author{
Von \\ A. Neumann und M. Lewandowsky.
}

(Aus der I. chirurgischen Abteilung des Krankenhauses Friedrichshain, Berlin [Direktor Prof. A. Neumann].)

\section{(Eingegangen am 20. Februar 1910.)}

\section{Hirngummi bei Lues hereditaria tarda.}

Antonie St., 17 Jahre alt.

Pat stammt angeblich aus gesunder Familie. Sie hatte als kleines Kind Masern, sonst aber keine Kinderkrankheiten gehabt. Sie war aber von früher Jugend an bleichsüchtig, schwerhörig und nierenleidend. Ihre Menses hat sie seit dem 14. Lebensjahre, aber immer unregelmäßig. Sie war das erstemal in unserem Krankenhause 1907, und zwar lag sie 4 Wochen wegen ihres Nierenleidens auf der Abteilung von Prof. Krönig.

Nach ihrer Entlassung entwickelte sich bei der Kranken an der rechten Seite des Halses, sowie in der rechten Achselhöhle Geschwülste, die spontan gar nicht, auf Druck nur wenig empfindlich waren. Diese Geschwülste wurden während eines zweiten Krankenhausaufenthaltes auf der chirurgischen Abteilung im Juli 1907 exstirpiert und erwiesen sich als derbe, mit der Umgebung schwartig verwachsene Drüsengeschwülste, die bei der Untersuchung durch Prof. Pick die unverkennbaren Zeichen der Tuberkulose darboten: durchgehends typische verkäsende Tuberkulose mit Riesenzellen. Sonst bot die Kranke damals außer einem chronischen Mittelohrkatarrh an beiden Seiten mit eitrigem Ohrenfluß keine Zeichen der Tuberkulose dar. Sie wurde nach dreiwöchentlichem Krankenhausaufenthalt mit geheilten Operationswunden nach Blankenburg entlassen.

ISie fand dann das drittemal bei uns Aufnahme am 2. Oktober desselben Jahres (1907), und zwar wegen eines Geschwüres, das sich 'ganz allmählich in der alten Operationsnarbe an der rechten Halsseite gebildet hatte. Wir konstatierten (an dem anämischen, für ihr Alter sehr kleinen Mädchen) hierselbst ein etwa zweimarkstückgroßes Geschwür mit scharfen, wie ausgefressenen, sinuösen Rändern, die umgebende Haut war kupferrot, derb infiltriert. Der Grund wurde von nekrotischen, speckig schmierigen Massen gebildet, die offenbar im Bereiche des ebenfalls derb infiltrierten und verdickten M. sternocleidomastoideus gelegen waren.

Wir konnten jetzt über die Diagnose eines geschwürig zerfallenen Gummis des M. sternocleidomastoideus nicht im Zweifel sein. An beiden Ohren alte Otitis media mit reichlicher cholesteatomähnlicher Masse. Temperatur subfebril $38,0^{\circ}$. Puls 84-100. Die Kranke wurde am 14. Oktober 1907 an dem wissenschaftlichen Abend unseres Krankenhauses vorgestellt (Neumann).

Bereits an demselben Abende klagte das Mädchen mit einem Male über sehr schlechtes Sehen und zunehmende Kopfschmerzen. Wir konstatierten eine mäßige 
rechtsseitige Internuslähmung und eine Erweiterung der rechten Pupille gegenüber der linken. Beide Pupillen reagierten gut. Dazu wurde durch den hinzugezogenen Augenarzt eine hochgradige Stauungspapille und zentrale glänzende kleine Flecken in der Retina konstatiert. Der Vortragende sprach damals schon die Vermutung aus, daß vielleicht neben der gummösen Veränderung des M. sternocleidomastoideus auch ein Hirngummi bei dem Mädchen vorliege. Diese Vermutung wurde gestützt einmal durch weitere anamnestische Erhebungen und zweitens durch den weiteren Krankheitsverlauf.

Anamnestisch konnten wir nämlich noch in Erfahrung bringen, daß die Kranke sohon Wochen vorher vielfach an Kopfschmerzen und Schwindelgefühl gelitten, daß sie auf dem rechten Auge geschielt und doppelt gesehen habe.

Besserung am 15. und 16. Oktober über Tag.

Es war sofort eine antisyphilitische Kur eingeleitet worden. Doch nahmen die Hirnsymptome jetzt rapide zu. Die Kopfschmerzen wurden in der Nacht vom 16. zum 17. Oktober so stark, daß Patientin gellende Schreie ausstieß. Dazu trat wiederholtes Erbrechen, eine Parese zuerst des linken Facialis, dann der ganzen linken Körperseite und eine auffallend starke Herabsetzung der Sensibilität der linken Körperseite. Temperatur $37,4^{\circ}$. Puls sehr wechselnd unregelmäßig, im Mittel 76-84.

17. Oktober. Lumbalpunktion ergibt $170 \mathrm{~mm}$ Druck, der nach Ablassen von $1 \mathrm{ccm}$ klarer Flüssigkeit auf ca. $130 \mathrm{~mm}$ sinkt.

Pat. ist im Laufe der Nacht völlig a maurotisch geworden. Beide Pupillen sehr weit, die rechte reagiert nicht mehr, die linke noch wenig auf Licht. Starke Benommenheit. Dabei heftige Kopfschmerzen. Mehrmaliges Erbrechen. Leichte Nackensteifigkeit. Die Untersuchung des Nervensystems ergab ferner, soweit bei der Benommenheit eine Untersuchung möglich: Mäßige Hemiparese der linken Seite, die besonders im Gebiete des unteren Facialis und an der Hand vollkommen ist, während Arm und Bein noch etwas bewegt werden. Patellar- und Achillessehnenreflex links deutlich schwächer als rechts. Babinski nicht vorhanden, aber Fußsohlenreflex und Bauchdeckenreflex links nur sehr schwach. Auffallend ist die außerordentlich geringe, fast fehlende Reaktion bei schmerzhaften Reizen der ganzen linken Körperseite, während die gleichen Reize rechts erhebliche Schmerzäußerungen zur Folge haben. Von einer geordneten Sensibilitätsprüfung konnte keine Rede sein.

Die diagnostischen und therapeutischen Erwägungen, die wir angesichts dieser Situation anstellten, waren folgende:

Wir befanden uns gegenüber einem Falle, der nach einigen Tagen der Vorbereitung, in denen eine Stauungspapille festgestellt worden war, ganz plötzlich, im Verlaufe weniger Stunden, sich so verschlimmert hatte, daß 1. eine starke Benommenheit, 2. eine totale Amaurose eingetreten war, und bei dem sich 3. als Zeichen dieser Verschlimmerung Herdsymptome von seiten der linken Körperseite eingestellt hatten. Daß der zugrunde liegende intrakranielle Prozeß mit starkem Hirndruck einherging, daran konnte, angesichts der Stauungspapille - trotz des verhältnismäßig geringen Lumbaldruckes - kein Zweifel sein. Die Frage, ob überhaupt ein circumscripter, intrakranieller Prozeß vorliegt, war, wie in einer Reihe von ähnlichen Fällen, nicht mit Sicherheit zu beantworten. Denn diejenigen Fälle sind gerade in der Krankenhauspraxis - es ist kein Zufall, daß gerade Non ne sie besonders gewürdigt 
hat - zu häufig, in denen trotz der anscheinenden Lokalsymptome eine Lokalerkrankung fehlt. Der vorliegende Fall war aber so dringend, daß diese Eventualität praktisch vernachlässigt werden mußte. Es war vielmehr klar, daß eingegriffen werden mußte, vor allem, um eventuell von der Sehfähigkeit zu retten, was noch zu retten war, und ferner bestand in Anbetracht der dauernd wachsenden Benommenheit eine Indicatio vitalis. Der Mechanismus dieser bei raumbeschränkenden intrakraniellen Prozessen plötzlich eintretenden Verschlimmerungen ist nicht ganz klargestellt. In dem vorliegenden Falle ist es jedenfalls, wenn auch eine genauere neurologische Untersuchung vorher nicht stattgefunden hatte, wahrscheinlich, daß die Herderscheinungen, die sich nunmehr fanden, wenigstens in der zur Manifestation gekommenen Intensität, fast akut entstanden waren, trotz der Größe des später gefundenen Tumors. Denn die Kranke hatte noch am Tage vorher weder eine Spur motorischer Störungen, noch von Ataxie gezeigt, und letztere wenigstens wäre unbedingt zur Erscheinung gekommen, wenn die später so in den Vordergrund tretenden sensiblen Störungen schon vor der akuten Verschlimmerung bestanden hätten.

Die Lokalisation des zugrunde liegenden Prozesses konnte angesichts der Benommenheit keine exakte sein. Geleitet wurden wir durch die auch in der Benommenheit auffällige Herabsetzung der Sensibilität der linken Seite, und deren Mißverhältnis zu der verhältnismäßig geringen motorischen Parese, wenn wir den Herd hinter der Zentralfurche annahmen und speziell auf die hintere Zentralwindung eingingen. Auch die Differenz der Pupillen und die Internusparese rechts konnte auf das Gebiet der mittleren Schädelgrube hinweisen.

Was die Art des Herdes betraf, so erwarteten wir von vornherein in erster Linie ein Gummi zu finden angesichts des gummösen Prozesses am Halse. Möglich wäre freilich auch ein Tuberkel (vorhergegangene Exstirpation sicher tuberkulöser Lymphdrüsen) gewesen und auch ein Hirnabsceß (doppelseitige alte Otitis), indessen sehr viel weniger wahrscheinlich.

Operation. 17. Oktober. (Neumann.) Trepanation im Bereich des rechten Scheitelbeins unter Bildung eines gestielten Haut-Periost-Knochenlappens, der, nach oben leicht abgerundet, unten etwa $21 / 2$ bis $3 \mathrm{~cm}$ oberhalb des Ohransatzes mit horizontaler, ca. $6 \mathrm{~cm}$ breiter Basis entspringt.

Als Zentrum wurde die Stelle gewählt, von der aus am ehesten ein Druck auf den hinteren Teil der inneren Kapsel erwartet werden konnte, eine Stelle, welche etwa der hinteren Zentralwindung entsprach.

Die freigelegte Dura wölbt sich stark in die über fünfmarkstückgroßen Knochen links vor, ist prall gespannt und zeigt keine Pulsation. Die Dura verdickt und sulzig getrübt. Unter ihr schimmert namentlich im vordern Teil der Lücke eine gelbliche Substanz hervor. Die Dura, kreuzweis eingeschnitten, erweist sich mit den unter ihr gelegenen Schichten locker verbacken; bei ihrer Ablösung quillt an einer Stelle spärlich Cerebrospinalflüssigkeit hervor. Die gelb verfärbte Sub- 
stanz zeigt eine homogene Struktur und steht in ihrer Konsistenz etwa zwischen Knorpel- und Bindegewebsfestigkeit. Nach hinten grenzt sich diese Masse deutlich gegen anscheinend normale Hirnsubstanz ab, und es gelingt, sie hisr stumpf aus letzterer herauszulösen. Weiteres stumpfes Vorgehen mit dem Finger löft schließlich einen gut apfelgroßen, als Gummi anzusprechenden Tumor aus seiner Umgebung heraus. Während seine hintere Grenze dem Auge sichtbar bleibt. reicht er nach vorn und unten unter den Schädelknochen herunter. Normale Gehirnsubstanz sieht man nur spärlich im hinteren oberen Quadranten der Trepanationsöffnung. Die Gyri scheinen abgeplattet.

Nach Enucleation des Tumors stellt sich alsbald Gehirnpulsation ein. Kleine parenchymatöse Nachblutung. Versorgung der Wunde. Fixation des Knochenlappens an seiner alten Stelle.

Der Tumor erwies sich mikroskopisch als Gummi (Pick).

18. Oktober. Pat. hat die Operation gut überstanden. Kopfschmerzen haben aufgehört. Temperatur normal. Puls ca. 100. Kein Erbrechen. Benommenheit erheblich geringer. Pupillen etwas enger. Sehvermögen gleich Null. Die Parese der linken Körperseite ist erheblich verstärkt.

Der Heilungsverlauf war ein ungestörter. Ein Hirnprolaps bildete sich ailmählich zurück, die Wunde war am 12. Dezem ber fast ganz vernarbt. Der Knochenlappen heilte spüter fest ein.

Die Benommenheit war etwa 2 Tage nach der Operation völlig verschwunden.

Das Sehvermögen schien sich in den ersten Wochen nach der Operation auf dem rechten Auge nicht unerheblich zu bessern, während es auf dem linken völlig verloren blieb. Am 5. November ist notiert, daß die Pat. die Utberschriften von Zeitungen richtig lesen, auch 1 Gegenstände und Finger richtig erkennen bzw. zählen konnte.

Fin Später aber, mit der Ausbildung einer Atrophie des Sehnerven, erlitt das Sehvermögen eine erhebliche abermalige Einschränkung, so daß die Pat. definitiv nur ein kleines Gesichtsfeld nach oben außen behalten hat, mit dem sie nicht imstande ist, sich im Raum zu orientieren, und auch nicht zu lesen.

Utber die cerebralen Ausfallserscheinungen wird unten berichtet.

Eine Übersicht über den Verlauf des Falles ergibt, daß die vor der Operation angestellten diagnostischen und therapeutischen Erwägungen sich als zutreffend erwiesen haben. Die Lokalisation mit der Direktive, auf die hintere Zentralwindung einzugehen, war richtig. Nach der Lage der Trepanationsöffnung muß der Tumor wohl der hinteren Zentralwindung bis dicht heran an die vordere entsprochen haben. Es ist aber kein Zweifel, daß die Ausfallserscheinungen, die der Tumor gemacht hat, und die zum größten Teil dauernd bestehen blieben, durch seine Ausdehnung in die Tiefe, d. h. nach der inneren Kapsel zu, bedingt waren, die Ausdehnung an der Oberfläche war zu gering.

Bestätigt hat sich weiter die Wahrscheinlichkeitsdiagnose dè Art der Herderkrankung als Gummi. Die Notwendigkeit der Operation eines Gehirng um mi gehört zu den sehr großen Seltenheiten. Oppenheim ${ }^{1}$ ) hat sich noch in keinem Falle von Hirnsyphilis zur Empfehlung einer operativen Behandlung entschließen können, erkennt

1) Lehrbuch, 5. Aufl. S. 1193. 
aber gewisse Indikationen an, wie denn auch von einer Reihe von Autoren bei luetischen Erkrankungen auch bei zutreffender Diagnose operiert worden ist $\left.{ }^{1}\right)$. Friedländer und Schlesinger stellen als Indikationen auf: 1. Stationärer Befund nach antisyphilitischer Behandlung; 2. Progredienz der Erscheinungen trotz eingeleiteter Behandlung, wenn eine Indicatio vitalis besteht; 3 . Trotz antiluetischer Behandlung bestehende $\mathbf{J}$ a ckson sche Epilepsie, auch wenn die früheren Tumorsymptome inzwischen geschwunden sind. Die zweite Indikation traf in unserem Falle zu. Daß ein Gummi auf Grundlage einer Lues hereditaria tarda operiert worden ist, dürfte bisher nicht bekannt sein. Gehören doch einzelne circumscripte Gummigeschwulste am Gehirn, ohne anderweitige luetische Erkrankungen insbesondere der Gefäße, bei der, hereditären Lues schon zu den allergrößten Seltenheiten ${ }^{2}$ ).

Der Erfolg der Operation war in bezug auf die Beseitigung der Lebensgefahr ein vollständiger und rascher. Das Sehvermögen hat sich trotz der erst wenige Stunden dauernden Amaurose nur in ganz geringem $\mathrm{MaBe}$ wiederherstellen lassen, und zwar erscheint es bemerkenswert, daß eine weitergehende anfängliche Besserung wohl unter dem Einfluß der Organisation des entzündlichen Exsudates (neuritische Atròphie) später wieder erheblich zurückgegangen ist.

Was nun die lokalen Ausfallssymptome betrifft, so machen sie den vorliegenden Fall auch in allgemein pathologischer Beziehung zu einem bemerkenswerten. Die Symptome der motorischen Parese, die durch die Operation zunächst verstärkt worden waren, gingen sehr schnell zurück, schon wenige Wochen nach der Operation war auch nicht die Spur einer motorischen Parese mehr nachzuweisen ${ }^{3}$ ), und zurück blieben nur die schweren Sensibilitätsstörungen, die von Anfang an die Lokalisation gegeben hatten.

Am 20. November 1907 ist notiert: Völlige Aufhebung des Muskelsinnes derart, daß auch maximale Exkursionen im Fuß- und Handgelenk, in den Zehenund Fingergelenken nicht wahrgenommen werden. Auch in den proximalen Gelenken ist die Störung eine sehr hochgradige, wenn auch nicht so vollkommene, wie in den distalen.

Gleichschwere Gegenstände werden in der linken Hand immer als schwerer empfunden wie in der rechten.

Berührungen werden auf der ganzen linken Seite nicht gefühlt, ebenso wenig leichter Druck. Es gelingt überhaupt nicht, mit Sicherheit eine Stärke

1) Vgl. bei Nonne: Syphilis und Nervensystem, 2. Aufl.; Berlin 1909, S. 599.

2) Vgl. Bresler: Erbsyphilis und Nervensystem. Schmidts Jahrbücher, Bd. 282 und Nonne 1. c.

3) Auch Babinskischer Reflex, Fußklonus usw., die zunächst vorhanden gewesen waren, verschwanden sehr bald. Der Fußsohlenreflex links war sehr abgeschwächt, der Bauchdeckenreflex kaum auszulösen. Diese Abschwächung der Hautreflexe darf aber im vorliegenden Falle wohl als Folge der Sensibilitätsstörung gedeutet werden. 
des Druckes auszuprobieren, die mit Bestimmtheit als Druck und nicht zugleich als Schmerz empfunden wïrde.

Ebenso werden Wär merei ze nur dann empfunden, wenn sie zugleich Schmerz verursachen. Dagegen wird ein mit $\mathrm{Eis}$ gefülltes Reagensglas nach längerem Liegen gelegentlich als kalt angegeben.

Schmerz wird nur bei länger dauernden Reizen empfunden. Kurze Stiche werden überhaupt nicht wahrgenommen. Dagegen wird längeres Kneifen unangenehmer und schmerzhafter empfunden als auf der gesunden rechten Seite. Die Lokalisation dieser schmerzhaften Empfindungen ist sehr schlecht.

Die Sensibilitätsstörung erstreckt sich auf die ganze linke Körperseite, hellt sich gegen die Mittellinie zu etwas auf, betrifft auch die Schleimhäute des Gesichts (der Cornealreflex ist intakt). Geschmackstörungen fehlen.

21. Januar 1909. Muskelsinn fast unverändert. Bewegungen in den Gelenken der linken Körperseite nur dann wahrgenommen, wenn sie schnell und wiederholt ausgeführt werden.

Leichte Berührungen und leichter Druck werden nicht empfunden.

Stärkerer Druck wird als solcher empfunden an der Stirn.

Am Arm muß der Druck noch stärker sein als an der Stirn, um als solcher empfunden zu werden. Manchmal wird unmittelbar, trotzdem mit ganz stumpfen Gegenständen berührt wird, angegeben: ,es piokt". Immer genügt eine kleine Verstärkung des Druckes, auch wenn man mit der Fingerkuppe drückt, um das Gefühl des Druckes in das des schmerzhaften Piekens übergehen zu lassen. Wenn man eine Hautfalte abhebt und zusammendrückt, so gehört ganz starker Druck dazu, um die Pat. überhaupt etwas empfinden zu lassen. und dann ist die Empfindung sofort die des schmerzhaften Kneifens oder Piekens. Gibt mit Bestimmtheit an, daß die Empfindung des Druckes, die sie auf der rechten normalen Körperseite hat, ihr links fehlt.

Temperatur. Am Arm wird Eiswasser zuerst als sehr kalt bezeichnet, bei Vergleich mit der rechten Seite als kälter und unangenehmer als auf dieser gesunden Seite; dabei ist die Latenzzeit links deutlich verlängert. Nach einigen Prüfungen hört die Empfindung kalt am linken Arm überhaupt auf und kehrt erst nach einigen Minuten wieder.

An der linken Hand wird Eiswasser überhaupt nicht als kalt empfunden. Am Bein analoges Verhalten wie am Arm: Hyperästhesie bzw. Hyperalgesie, schnelle Ermüdbarkeit, Abnahme der Empfindlichkeit gegen das distale Extremitätenende hin.

Im Gesicht wird Eiswasser immer als kalt wahrgenommen, wenn auch mit größerer Latenz und als unangenehmer, bzw. „,kälter" als auf der gesunden Seite.

Wasser bis $44^{\circ} \mathrm{C}$. wird an den linken Extremitäten noch nicht als warm wahrgenommen. Bei Wasser von $50^{\circ} \mathrm{C}$., das am rechten Arm sehr gut vertragen wird, links sehr lange Latenz (ca. 5 Sek.), dann plötzlich das Gefühl ,heiß“, ausdrücklich mit der Angabe, daß ,vorher gar nichts" war.

Im Gesicht wird schon eine Temperatur von $41^{\circ}$ als warm und zwar als wärmer wie auf der gesunden Seite empfunden, und eine Temperatur von $44^{\circ}$ löst links manchmal die Empfindung „heiß' aus, während sie rechts natürlich als warm empfunden wird.

Die Sohmerzempfindung unverändert.

25. Januar 1910. Der Zustand der Sensibilität hat sich im ganzen wohl etwas, aber unerheblich gebessert: nur bei sehr großen Exkursionen der Gelenke hat sie das dunkle Gefühl, daß etwas bewegt wird, kann die Lage der Glieder aber durchaus nicht angeben. Berührungen werden nach wie vor nicht wahrgenommen; dagegen ist der Schwell nnwert des Druckes, der als solcher empfunden wird, wohl etwas geringer geworden. Schmerzempfindung wie früher. Lokalisation dafür 
immer noch schlecht, aber doch besser als früher. Auch die Empfindlichkeit Temperaturreizen, insbesondere Kälte, gegenüber besser. Insbesondere wird Wasser von $18^{\circ} \mathrm{C}$. überall als kalt angegeben, während Temperaturen von $48^{\circ} \mathrm{C}$. (mit Ausnahme des Gesichtes) noch immer nicht als warm empfunden werden.

Selbstverständlich ist von Stereognosis links keine Spur vorhanden.

Auf die Art der Sensibilitätsstörungen, die hier aufgezählt sind, und die im einzelnen manches Bemerkenswerte bieten (z. B. die Kombination von Hypästhesie mit Hyperalgesie), soll hier weiter nicht eingegangen werden. Es genüge die Registrierung.

Der Fall beweist jedenfalls von neuem die von Henschen, Dejerine, Long noch bestrittene, aber doch schon in Fällen von $R \approx y-$ mond, Dercum, Spiller, v. Monakow, v. Stauffenberg erhobene Tatsache, daß die Fasern für Sensibilität und Motilität nahezu völlig getrennt verlaufen müssen. Denn an eine Beteiligung des Thalamus kann in unserem Falle nicht gedacht werden, und es ist auch nach Lage des Tumors ausgeschlossen, daß eine Zerstörung der grauen Rinde in genügender Ausdehnung stattgefunden hat. Die Sensibilitätsstörung ist vielmehr zu beziehen auf die Zerstörung des Markes des Gyrus centralis posterior (vielleicht auch des Gyrus supramarginalis), wie eine solche in seinem Falle von v. Stauffenberg festgestellt wurde, und vielleicht reicht der Tumor bis in den retrolentikulären Abschnitt der inneren Kapsel. In bezug auf die völlige Verschonung der Motilität bei einer äußerst schweren und dauernden (jetzt $2 \frac{1}{2}$ Jahre) Sensibilitätsstörung ist unser Fall wohl der reinste der beobachteten.

Von sehr großem Interesse war uns nun, gerade in Anbetracht der Unversehrtheit der Motilität im engeren Sinne, die Beobachtung der als Folge der Sensibilitätsstörung auftretenden cerebralen Ataxie. Es erscheint uns nach dieser und auch nach einigen anderen analogen Beobachtungen, als wenn die cerebrale Ataxie besondere Eigentümlichkeiten hat, während von anderen, z. B. von Bickel angegeben wird, daß sie sich von der spinalen in nichts unterscheide. In unserer Beobachtung trat von Anfang an, trotzdem die Ataxie sehr stark war, das Unregelmäßige, Sakkadierte, das die spinale Ataxie charakterisiert, sehr zurück. Wenn man die Kranke z. B. aufforderte, mit dem Zeigefinger nach der Nase zu fassen, so geschah das nicht so, wie beim Tabiker, daß der Finger nach starken Schwankungen doch ziemlich in der Nähe der Nase anlangte, sondern der Finger fuhr fast geradewegs gegen die Stirn oder ins Auge. Die Abweichung in der Richtung und dem AusmaB der Bewegung war ganz unverhältnismäBig stark gegenüber der Unsicherheit der Ausführung, und so war es auch bei allen anderen Bewegungen. Fordert man die Kranke auf, mit ihrer linken Hand berührte Stellen der rechten Körperseite zu zeigen, so muß man sich hüten, die so entstehenden Fehler als Lokalisationsfehler zu nehmen, 
sie sind nur der Ausdruck der vollkommen verloren gegangenen Präzision in der Richtung der zeigenden Hand.

Alle Bewegungen werden ferner dem Sinne nach richtig (d. h. ohne eine.Spur von Apraxie), aber in sehr großem Ausmaße ausgeführt. Pat. kann überhaupt auch nicht im entferntesten so kleine Bewegungen mit dem Arm oder den Fingern der linken Seite ausführen, wie mit denen der rechten.

Besonders merkwürdig war das Gehen trotz der fast vollkommenen Anästhesie des Beines. Es fand sich niemals die Spur eines schleudernden Ganges, niemals auch ein Schwanken im Sinne des Romberg schen Phänomens, im Anfang knickte die Kranke auf dem kranken Bein einfach zusammen, später fing sie an, es langsam vorzuschieben, und dann wurde der Gang fast normal. Über die Art, wie sie das fertig brachte, war die Kranke nicht imstande, genügend Auskunft zu geben. Sie sagte, sie fühle den Boden nicht ordentlich, sie ginge wie auf einem Stelzbein, das Bein wäre so schwer, sie müsse aufpassen, daß es nicht verkehrt gehe, weil es sich manchmal von selbst drehe, aber es war ihr offenbar selber unklar, wie sie mit dem Bein, an dem sie nichts fühlte. gehen konnte. Es spricht das dafür, daß die Ausführung der Koordination beim Gehen in hohem Maße den niederen Zentren, insbesondere wohl dem Kleinhirn, zufällt. Auf dem linken Bein allein zu stehen vermag die Pat. auch heute übrigens noch nicht.

Die Ataxie hat ferner im Laufe der Jahre überhaupt - auch an den oberen Extremitäten - erheblich und in unverhältnismäßig höherem Maße als die Sensibilitätsstörungen abgenommen, und das, trotzdem der Gesichtssinn doch dauernd so gut wie völlig ausgeschaltet war. Es muß diese Rückbildung wohl als eine Anpassung durch einen Ersatz seitens anderer Zentren erklärt werden. Jetzt ist der Gegensatz zwischen der Intensität der Sensibilitätsstörung und der verhältnismäßig geringfügigen Bewegungsstörung in der Tat sehr auffallend, ein Gegensatz, der für Thalamusschädigungen von Dejerine betont wird. Es ist das natürlich ein Beweis, daß die sensible Regulierung der Bewegung zum sehr großen Teil unterhalb des Großhirns vor sich geht, und daß die bewußte Empfindung, die ja bei Tabischen trotz schwerer Ataxie doch nur in geringem Maße gestört zu sein braucht, nicht einmal die koordinatorische Tätigkeit der niederen Organe ganz ersetzen kann.

Erwähnt sei noch, daß eine wesentliche Hypotonie auch zu den Bestandteilen der cerebralen Ataxie gehört.

\section{Metastatischer HirnabsceB.}

Frau Sch. ist am 25. August 1909 wegen eines rechtsseitigen Empyems des Antrum. Highmori von Dr. Jansen operiert worden. Es soll sich viel 
Eiter entleert haben. Nach achttägigem Aufenthalt in der Klinik wurde sie am 1. September entlassen und hat sich dann zu Hause wohlgefühlt. Am 15. September begann ein Erysipel, das von der Nase aus auf die rechte Kopfseite sich ausbreitete und nach 8 Tagen, am 22 . September, abgeheilt war.

Am 27. September stellten sich halbseitige Krämpfe und Lähmungserscheinungen der linken Körperseite ein. Am 29. September wurde sio dem Krankenhaus zugeführt.

Der aufnehmende chirurgische Assistenzart stellte die Diagnose „Hirnabsceß“ und wies deswegen die Kranke sofort der chirurgischen Abteilung zu.

Temperatur $37,8^{\circ}$. Puls 104.

Pat. ist leidlich klar, gibt Auskunft. Linksseitige starke Parese, die den Facialis, Arm und Bein betrifft. Babinski + . Patellarreflex links stärker als rechts, kein Fußklonus. Bauchdeckenreflex links + . Keine deutlichen Sensibilitätsstörungen.

Rechte Pupille etwas weiter als die linke, eine Spur von Ptosis rechts. Pupillen reagieren. Die Gefäße der rechten Papille stark gefüllt, der Rand der Papille ein wenig unscharf, keine Niveaudifferenz.

Kurz nach der Einlieferung bekommt Pat. einen Krampfanfall klonischer Art, der links im Facialisgebiet beginnt, sich dann sehr schnell auf die linke und auch auf die rechte Körperseite ausbreitet. Während des Anfalls sind die Augen nach links gerichtet, nach dem Anfall besteht eine Deviation nach rechts. Schon einigee Sekunden vor Beginn der Zuckungen wird Pat. benommen. Die Dauer des Anfalles betrug einige Minuten.

Pat. hat in der Nacht 25 Anfälle, deren Dauer auch allmählich zunimmt, so daß die Pat. einmal 1/2 Stunde ununterbrochen krampfte.

Am 30. September war Pat. völlig benommen, fast komatös. Die Diagnose „Hirnabsce $B^{\prime \prime}$ erschien fast völlig sicher und am 30. September mittags wurde zur Operation geschritten.

$\mathrm{DaB}$ der Hirnabsceß in der Nähe der motorischen Region (vordere Zentralwindung) liegen mußte, war wohl sicher. Wegen der schon zu Beginn der Krämpfe eintretenden Deviation der Augen, wurde daran gedacht, daß der Herd sich eher etwas nach vorn von der vorderen Zentralwindung befinde.

Operation (Neumann), da Pat. völlig benommen ist, in leichter Chloroformnarkose.

Bildung eines Haut-Periost-Knochenlappens rechts. Hintere Begrenzung senkrecht nach oben vom rechten Gerhörgang, vordere Begrenzung Haargrenze. Basis zwei Querfinger oberhalb des oberen Ohrumfanges. Obere Grenze ein Querfinger von der Medianlinie.

Nach Freilegung der Dura, die deutlich pulsiert, stößt man bei vorsichtiger Punktion unmittelbar unter der Dura, und zwar in der hinteren Hälfte der freigelegten Fläche, auf Eiter. Die Dura wird incidiert; es entleeren sich etwa zwei Teelöffel Eiter vom hinteren Umfang her, aus einem zum Teil subdural, zum Teil nach hinten in der Substanz des Gehirns selbst liegenden Abscesse. Nach Entleerung des Eiters Einführung eines Gazestreifens in den in der Hirnsubstanz liegenden Teil des Abscesses. Einlage eines feinen Drains unter die Dura. Verband.

Am Abend des Operationstages erhebliche Aufhellung des Bewußtseins. Die Krämpfe haben sich nach der Operation noch dreimal wiederholt. Schon am Abend kann sie das linke Bein etwas heben.

1. Oktober ein Krampfanfall. Sensorium fast frei. Temperatur bis $37,7^{\circ}$ Puls 116-124.

5. Oktober Verbandwechsel. Temperatur normal. Puls 100-108.

Keine Krämpfe seit dem 1. Oktober. 

Kraft.

Pat. bewegt die Glieder der 1. Seite ziemlich ausgiebig, wenn auch mit geringer

2. Oktober. Heut $38,6^{\circ}$. Puls 120. Pat. klagt über Kopfschmerz. Verbandwechsel.

In den nächsten Tagen noch einige Temperatursteigerungen, die aber den im ganzen günstigen Verlauf nur etwas verzögerten.

Am 2l. Oktober wurden die Streifen entfernt.

Am 30. Oktober steht Pat. auf.

Am 11. November wird Pat., nachdem sich noch einige Knochensequester abgestoßen hatten, mit Verband entlassen.

Von Ausfallserscheinungen seitens des Nervensystems bestand damals nur eine geringe Beweglichkeitsbeschränkung des Armes (besonders der Abduktion), eine geringe Sensibilitätsstörung der linken Seite, und eine sehr starke Abweichung der Zunge nach links. Eine leichte Facialisparese war kombiniert mit einer geringen aber deutlichen Neigung zur Kontraktur in demselben Gebiet.

Pat. ist dauernd in unserer Beobachtung geblieben. Noch zweimal haben sich kurze Reihen von ihrer Beschreibung nach Jacksonschen Anfällen eingestellt, bei denen sie das Bewußtsein nicht verlor, und die beide Male nach einigen Tagen mit der Ausstoßung eines kleinen Knochensequesters endigten.

Pat. befindet sich jetzt (März 1910) völlig wohl, ist arbeitsfähig, und versieht ihren Haushalt wie früher. Am längsten hat sie eine Sensibilitätsstörung in der linken Hand gestört. Sie hatte das Gefühl von Taubheit in der Hand und objektiv ließ sich eine geringe Herabsetzung aller Qualitäten (mit sekundärer Stereognosis) feststellen, die jedoch in Rückbildung begriffen ist. Die Motilität ist völlig wiederhergestellt, die Differenz der motorischen Kraft nur noch sehr gering. Auch die Deviation der Zunge ist nur noch gering. Kein Fußklonus, kein Babinski.

Die Diagnose eines metastatischen Hirnabscesses war ja in diesem Falle nicht schwer. Das plötzliche Einsetzen von einseitigen Krämpfen nach U̇berstehen eines Erysipels und nach Operation eines Empyems der Highmorshöhle war ein genügender Hinweis. Ob der Absceß auf das Empyem oder erst auf das Erysipel zurückgeht, ist mit Sicherheit kaum zu entscheiden. Wie weit das Erysipel nach dem behaarten Kopf fortgeschritten war, ist nicht festgestellt worden. Daß es sehr ausgedehnt gewesen ist, ist nach den Berichten des Hausarztes nicht anzunehmen und auch nicht wahrscheinlich, da Pat. nach 8 Tagen wieder vollkommen hergestellt war. Wir neigen daher mehr dazu, die lange bestehende Highmorshöhleneiterung als direkte Ursache des Abscesses anzunehmen. Die Lokalisation war in Anbetracht des Vorhandenseins einseitiger Krämpfe im groben ja auch gegeben. Eine genaue Bestimmung der Stelle, wo der Absceß gelegen war, war bei der Operation nicht möglich, da Eiter sofort gefunden und darauf natürlich von einer weiteren Entblößung des Gehirns Abstand genommen wurde. Indessen dürfte sich der Absceß kaum hinter die Zentralfurche erstreckt haben. Eigentümlich war die Lage des Abscesses halb unter der Dura und halb im Gehirn. Wahrscheinlich dürfte der ursprünglich intracerebrale, aber oberflächlich liegende Absceß nach außen durchgebrochen sein, nachdem vorher Verklebungen der Dura mit 
dem Gehirn zustande gekommen waren. Bemerkenswert ist der volle Erfolg, trotzdem die Operation in schwerem Koma vorgenommen wurde.

Die Anzahl der operierten metastatischen Hirnabscesse ist eine ganz außerordentlich geringe. Nach $O p$ penheim und Cassirer1) ist bei metastatischem Hirnabsceß noch in keinem Falle dauernde Heilung eingetreten, und somit wäre der berichtete Fall der erste, durch Operation geheilte Fall. Denn es liegt gar kein Anlaß vor, an der Definitivität und Vollkommenheit der Heilung zu zweifeln. Auch in unserem Material ist der berichtete der erste geheilte, aber nicht der erste chirurgisch behandelte Fall eines metastatischen Hirnabscesses. Es sind noch zwei andere Fälle von Hirnabscessen chirurgisch behandelt worden. In dem einen wurde ein Absceß des Parietallappens gefunden und eröffnet. Die Patientin überlebte den Eingriff auch einige Monate, ging aber schließlich marantisch zugrunde. In dem anderen Fall wurde die Trepanation an der richtigen Stelle gemacht (über dem Armzentrum), die Nadel glitt aber mehrere Male an dem im Marklager gelegenen $A b s c e ß a b$, wie das auch anderen schon passiert ist ${ }^{2}$ ), so daß der Schädel ohne Ergebnis wieder geschlossen wurde. Jeder diagnostizierte metastatische Hirnabsceß soll chirurgisch angegriffen werden auf die Gefahr hin, daß multiple Abscesse vorhanden sind, und aus diesem Grunde die Operation nutzlos ist. Aber die multiplen Abscesse bilden, in unserem Material, das wir zum Teil intra vitam ${ }^{3}$ ), zum Teil auch erst auf dem Sektionstisch sahen, die große Minderzahl gegenüber den unilokulären. Es widerspricht das der allgemeinen Annahme. Es scheint aber, als wenn die Angaben über Vorkommen und Symptomatologie des metastatischen Hirnabscesses, wie sie z. B. von Oppenheim und Cassirer zusammengefaßt werden, sich wesentlich auf diejenigen Fälle beziehen, in denen die Hirnabscesse als Teilerscheinung eines allgemeinen pyämischen, mit hohem Fieber, Schüttelfrösten usw. einhergehenden Prozesses, z. B. bei Endocarditis ulcerosa, auftreten. In diesen Fällen die in der unten gegebenen kleinen Statistik nicht mitgerechnet sind, sind die Hirnabscesse in der Tat meist - nicht immer - multipel, hier wird aber eine operative Behandlung von vornherein wenig Chancen haben. Sonst sollte sie in allen Fällen versucht werden.

1) Oppenheim u. Cassirer, Hirnabsceß, 2. Aufl. 1909, S. 263.

2) Vgl. darüber Oppenheim u. Cassirer, l. c., S. 255.

3) Der eine von uns (L.) hat im ganzen 5 metastatische Hirnabscesse (ohne allgemeine pyämische Erscheinungen) intra vitam zu sehen Gelegenheit gehabt. Von diesen waren vier einzelne, nur ein Fall multipel. Von den 4 einherdigen wurden die obigen 3 zur Operation gebracht, in einem wurde die Fehldiagnose auf Tuberkel mit tuberkulöser Meningitis gestellt. Es ist dabei zu bemerken, daß in dieser Statistik die Fälle, in welchen auch nur die entfernte Möglichkeit einer traumatischen Entstehung (Trauma ohne Kopfwunde in der Anamnese, alte Narbe der Kopfhaut) gegeben war, nicht mitgerechnet sind. 